\title{
Therapeutic Applications of Vaccine Nanoformulations
}

\author{
Mohanty C* \\ Laboratory for Nanomedicine, Institute of Life Sciences, Nalco Square, \\ Chandrasekharpur, Bhubaneswar, Orissa, India
}

*Corresponding author: Chandana Mohanty, Laboratory for Nanomedicine, Institute of Life Sciences, Nalco Square, Chandrasekharpur, Bhubaneswar, Orissa, India, Email: chandanamohanty@gmail.com

\section{Mini Review \\ Volume 2 Issue 5}

Received Date: April 10, 2018

Published Date: April 25, 2018

\begin{abstract}
Nanotechnology in the field of vaccines delivery encompass various nanocarriers that have variety of compositions, sizes, and surface properties. These vaccines loaded or decorated on to the surface of nanocarriers help to induce both humoral and cell-mediated immune responses. This review discussed recent advances in nanocarrier-based vaccine delivery systems including polymeric nanoparticles, liposomes, micelles, ISCOMs and virus-like particles (VLPs) to augment immunity against various diseases.
\end{abstract}

Keywords: Infectious Diseases; Vaccines

\section{Introduction}

Vaccination is one of the most effective public health interventions that had a key impact on the management of infectious diseases [1,2]. It is a preparation of small amount of a biological agent that resembles a microorganism. Most of the vaccines have been developed using live attenuated organisms, killed whole organisms or inactivated toxins (toxoids). Upon administration, it helps to develop immunity to a particular disease by stimulates the immune system of our body to recognize the agent as foreign and ultimately destroy it and remember it. It will be in memory in order to easily recognize further and able to fight against any of these microorganisms that it later encounters [3,4]. Conversely, there are many infectious diseases for which the development of an effective vaccine has been restricted. In many cases the failure to devise vaccines is a result of the failure of vaccine candidates to evoke appropriate immune responses $[5,6]$.
As a consequence, interest has been directed toward the utility of nanotechnology delivery systems for delivery of vaccines $[7,8]$. In perticular, nanoparticles (NPs) and microparticles have been as an important strategy to deliver vaccines, as well as biomacromolecules such as proteins or DNA [9-11]. The vaccine antigen is either encapsulated within or decorated on to the surface of the NPs. On encapsulation of the antigen the NPs protects the vaccines from rapid degradation upon injection thereby induce a sustained immune response. By conjugation of antigens onto NPs be able to allow presentation of the antigen to the immune systems in much the same way that it would be presented by the pathogen, thereby provoking a similar response $[4,5,11]$. Furthermore, NPs prepared from various composites facilitate not only site directed delivery of immunogen but also the sustained release of antigens to maximize exposure to the immune system. In recent years, many researchers have attempted to design the NPs to deliver vaccines to evoke appropriate immune responses. In 
recent years the development of various novel vaccine delivery systems using these nanotechnology will certainly be effective on providing potent antigen-specific humoral and cellular immune responses [3,11-13]. Moreover, recently nanotechnologies have been afforded various novel engineered vaccines against a range of infectious diseases.

\section{Limitation Associated with Vaccine Delivery}

A major drawbacks of using live vaccines comprise a serious risk of relapsing back to their virulent form and further its intrinsic instability making difficult to deliver. In addition, killed or inactivated whole organism vaccines and toxoid vaccines most of the time induces a weaker immune response and usually necessitate several doses [1,14-19]. To this end, adjuvants have been used in a view to protect antigens from degradation however, its application usually depends on its nature $[3,4,12]$. For example some adjuvants are aluminium based but these can induce local reactions and may fail to generate strong cell-mediated immunity. Also researches confirmed that model protein antigens are actually destabilized by traditional aluminum salt adjuvants. Vaccines based on recombinant DNA and proteins were developed that afford more safety than traditional vaccines because these do not contain the whole pathogen in its native live or attenuated form for eliciting its associated disease, however, its application mostly associated with less immunogenic response. Moreover, vaccines meant for a lot of infectious diseases are still not developed or unavailable and their effectiveness is inadequate by their poor ability to cross biologic barriers to reach the specific sites $[3,4,12]$. To solve the above explained limitations, there is an urgent need for the development of a delivery systems for the next generation of vaccines.

\section{Nanotechnology in Vaccine Delivery}

To overcome the intricacy associated with the conventional vaccine delivery, the progress of nanotechnology offers an effective approach which could provide significant benefits to immunization. In this regard, application of nanotechnology for vaccine delivery has gained a lot of attention $[11,13]$. In nanotechnology based vaccine delivery system, the nanocarriers are either encapsulating the antigen within or decorated it on to the surface. On encapsulation of the antigen the NPs protects the vaccines from rapid degradation upon injection thereby induce a sustained prolonged immune response. By conjugation of antigens onto the surface of NPs, permit targeted presentation of the antigen to the immune systems in much the same way that it would be presented by the pathogen. Currently, the introduction of nanotechnology for vaccine delivery and targeting is likely one of the most exciting and clinically important applications for eliciting immunity. The advent of nanotechnology coupled with a better understanding of various issues involved in vaccine delivery afforded development of various novel delivery systems against various fatal diseases. Beside better delivery and protection to encapsulated antigens at the same time it facilitates better cellular uptake owing to its nanoscale in size [9,13]. For example, antigen delivered through polymeric NPs are reported to taken up by M cells very effectively in the nasal-associated lymphoid tissue (NALT) when administered nasally, and it serves as a potent vehicle for mucosal immunization [20]. In comparison to encapsulated antigen the surface adsorbed antigen offers improved stability and immunity $[4,10]$. In this regards, nanotechnology offers various type of nanoscale delivery systems such as liposome, solid lipid nanoparticles, polymeric nanoparticles, functionalized nanoparticles, dendrimers, metallic nanoparticles, etc. Such competent nanoparticulate delivery systems are so engineered with the hope that they can act as likely candidates to interact with biological barriers and transport the bioactive molecule without altering its antigenicity.

Currently, nanotechnology offers various vaccine delivery systems that have been employed for evoking immune responses includes polymeric nanoparticles, liposomes, micelles, ISCOMs. and Virus-like particles (VLPs) (Figure 1). The commonly used delivery system is the NPs mostly prepared from various biodegradable polymers. It is mostly prepared from a range of polymer including poly (lactic-coglycolic acid) (PLGA) or poly(lactic acid) (PLA) [11-13]. Moreover, its sustained release kinetics of antigen can be controlled by modulating the matrix components with various copolymer. Further, Liposomes are known to be an professional vehicle for vaccine delivery. It has a microscopic structure containing an aqueous core surrounded by phospholipid bilayer capable of encapsulating any protein or biomolecule [21]. Micelles are prepared when amphiphilic molecules spontaneously associate in aqueous medium to form core-shell structures or vesicles in which the hydrophobic core is surrounded by a shell of hydrophilic polymers [22]. It is reported to demonstrate increased vascular permeability thus these can be used as proficient delivery for antigen. Colloidal saponin containing micelles $(\sim 40$-nm cage-like particle) can be used as self-adjuvanting vaccine delivery systems and are collectively known as ISCOMs [23]. protein antigens (typically hydrophobic membrane 
proteins) can be trap in the matrix of ISCOM through apolar interactions. It is bodily negatively charged and has the tendency to interact with positively charged antigens through electrostatic interactions. ISCOMs have been shown to be highly immunogenic not only when injected but also when delivered through the oral rout [23]. VLPs prepared from non-infective viruses and these are formed of self-assembling nanoparticles (20-60 nm in diameter). VLPs has several epitopes on their surface and be similar to native virions. Its structure is capable of inducing stronger immune responses. They also can express one or several viral structural proteins in a recombinant heterologous system. It can induce protection not only against the virus of origin but also against heterologous antigens [24].

\section{Conclusion}

The nanotechnology based vaccine delivery systems have been established as an efficient antigen delivery systems to augment immunity against various diseases due to their small size, large surface area to mass ratio, stability, aptitude for surface modification, and their control rate of degradation to allow sustained release of the antigens. However, the future of nanotechnology based vaccine delivery systems will be highly dependent on the development of safe, non-toxic and nonimmunogenic nanocarriers. There are still safety concerns associated with the introduction of such particles to the human body.

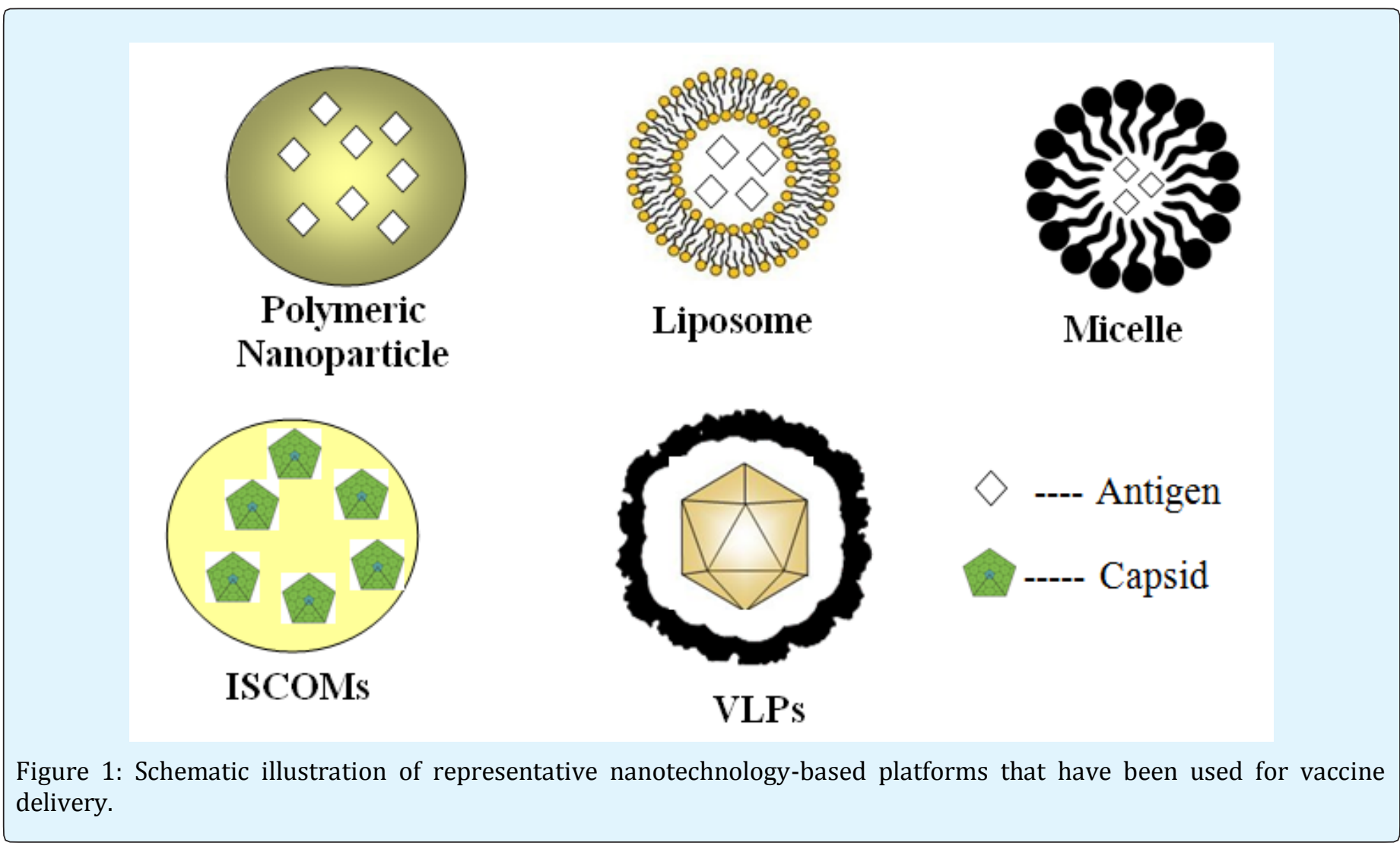

\section{References}

1. Bowen WS, Svrivastava AK, Batra L, Barsoumian H, Shirwan H (2018) Current challenges for cancer vaccine adjuvant development. Expert Rev Vaccines 17(3): 207-215.
2. Zheng Z, Diaz-Arevalo D, Guan H, Zeng M (2018) Noninvasive vaccination against infectious diseases. Hum Vaccin Immunother 6: 1-64.

3. Izumoto S (2012) Peptide vaccine. Adv Exp Med Biol 746: 166-177. 


\section{Virology \& Immunology Journal}

4. Platten M, Bunse L, Riehl D, Bunse T, Ochs K, et al. (2018) Vaccine Strategies in Gliomas. Curr Treat Options Neurol 20(5): 11.

5. Yamanaka R (2006) Novel immunotherapeutic approaches to glioma. Curr Opin Mol Ther 8(1): 4651.

6. Yamanaka R (2008) Cell- and peptide-based immunotherapeutic approaches for glioma. Trends Mol Med 14(5): 228-235.

7. Prasad S, Cody V, Saucier-Sawyer JK, Saltzman WM, Sasaki CT, et al. (2014) Polymer nanoparticles containing tumor lysates as antigen delivery vehicles for dendritic cell-based antitumor immunotherapy. Nanomedicine 7(1): 1-10.

8. Tran TH, Tran TTP, Nguyen HT, Phung CD, Jeong JH, et al. (2018) Nanoparticles for dendritic cell-based immunotherapy. Int J Pharm 542(1-2): 253-265.

9. Badiee A, Heravi Shargh V, Khamesipour A, Jaafari MR (2013) Micro/nanoparticle adjuvants for antileishmanial vaccines: present and future trends. Vaccine 31(5): 735-749.

10. Powell TJ, Palath N, DeRome ME, Tang J, Jacobs A, et al. (2011) Synthetic nanoparticle vaccines produced by layer-by-layer assembly of artificial biofilms induce potent protective T-cell and antibody responses in vivo. Vaccine 29(3): 558-569.

11. Zhao L, Seth A, Wibowo N, Zhao CX, Mitter N, et al. (2014) Nanoparticle vaccines. Vaccine 32(3): 327337.

12. Chesson CB, Zloza A (2017) Nanoparticles: augmenting tumor antigen presentation for vaccine and immunotherapy treatments of cancer. Nanomedicine (Lond) 12(23): 2693-2706.

13. Zang X, Zhao X, Hu H, Qiao M, Deng Y, et al. (2017) Nanoparticles for tumor immunotherapy. Eur J Pharm Biopharm 115: 243-256.

14. Buteau C, Markovic SN, Celis E (2002) Challenges in the development of effective peptide vaccines for cancer. Mayo Clin Proc 77(4): 339-349.
15. Dalgleish A (2007) Overcoming technical challenges in the development of cancer vaccines. IDrugs 10(7): 463-467.

16. Paul S, Acres B, Limacher JM, Bonnefoy JY (2007) Cancer vaccines: challenges and outlook in the field. IDrugs 10(5): 324-328.

17. Pejawar-Gaddy S, Finn OJ (2008) Cancer vaccines: accomplishments and challenges. Crit Rev Oncol Hematol 67(2): 93-102.

18. Scanlan MJ, Jager D (2001) Challenges to the development of antigen-specific breast cancer vaccines. Breast Cancer Res 3(2): 95-98.

19. Ward S, Copier J, Dalgleish A (2008) Technical challenges facing therapeutic cancer vaccines. Curr Opin Drug Discov Devel 11(2): 168-177.

20. Date Y, Ebisawa M, Fukuda S, Shima H, Obata Y, et al. (2017) NALT M cells are important for immune induction for the common mucosal immune system. Int Immunol 29(10): 471-478.

21. Marasini N, Ghaffar KA, Skwarczynski M, Toth I (2017) Liposomes as a Vaccine Delivery System. Micro and Nanotechnology in Vaccine Development 2:12-25.

22. Li C, Zhang X, Chen Q, Zhang J, Li W, et al. (2018) Synthetic Polymeric Mixed Micelles Targeting Lymph Nodes Trigger Enhanced Cellular and Humoral Immune Responses. ACS Appl Mater Interfaces 10(3): 2874-2889.

23. Garcia A, Lema D (2016) An Updated Review of ISCOMSTM and ISCOMATRIXTM Vaccines. Curr Pharm Des 22(41): 6294-6299.

24. Luo Y, Mohan T, Zhu W, Wang C, Deng L, et al. (2018) Sequential Immunizations with heterosubtypic viruslike particles elicit cross protection against divergent influenza A viruses in mice. Sci Rep 8: 4577. 